\title{
The near-nerve sensory nerve conduction in tarsal tunnel syndrome
}

\author{
SHIN J OH, HYUN S KIM, BASHIRUDDIN K AHMAD \\ From the Department of Neurology, University of Alabama at Birmingham, Birmingham, Alabama, USA
}

SUMMARY The near-nerve sensory nerve conduction in the medial and lateral plantar nerves was studied in 25 cases of tarsal tunnel syndrome. Sensory nerve conduction was abnormal in 24 cases (96\%) The most common abnormalities were slow nerve conduction velocities and dispersion phenomenon (prolonged duration of compound nerve action potentials). These two electrophysiological abnormalities are indicative of a focal segmental demyelination as the primary pathological process in tarsal tunnel syndrome.

In 1979, we published a method of obtaining sensory nerve conductions in the medial and lateral plantar nerves using the surface electrode, which was able to confirm the diagnosis of tarsal tunnel syndrome in $90 \%$ of cases.' For the past four years we have used the near-nerve needle sensory nerve conduction technique in diagnosis of this disorder. We report here our experience with this technique.

\section{Materials and methods}

The technique of the near-nerve needle sensory nerve conduction in the digital and interdigital nerves of the foot was described in detail in a previous report. ${ }^{2}$ Sensory compound nerve action potentials (CNAPs) were recorded orthodromically using the near-nerve needle and signal averaging techniques. The active needle recording electrode was inserted close to the medial plantar fascicle near the posterior tibial nerve in the ankle behind the medial malleolus above the flexor retinaculum. This site was chosen with the aid of a surface stimulating electrode. The adequacy of the needle position was determined by stimulating the nerve through the active electrode. When the great toe (digit I) was contracting minimally with less than $3 \mathrm{~mA}$ for a stimulus of $0.05 \mathrm{~ms}$ duration, the needle was considered to be adequately positioned. The reference needle electrode was placed subcutaneously at the same level as the active electrode at a transverse distance 3$4 \mathrm{~cm}$. The $I$ and $V$ digital nerves were stimulated with ring electrodes and various interdigital nerves with interdigital stimulating surface electrodes which were specially designed to stimulate two branches of the interdigital nerve

Address for reprint requests: Shin J Oh, MD, Department of Neurology, University of Alabama at Birmingham, University Station, Birmingham, Alabama 35294, USA.

Received 7 December 1984 and in revised form 1 March 1985. Accepted 8 March 1985. simultaneously. Each interdigital nerve was stimulated separately by placing the interdigital stimulating electrodes between the toes. Stimulus duration used was 0.05-0.1 ms. Supramaximal stimulation was assured by increasing it $25-30 \%$ beyond the maximal stimulation intensity when the CNAP was observed on each stimulus. The supramaximal stimulation intensity was usually at least three times the sensory threshold level and above $60 \mathrm{~mA}$ for a stimulus of $0.05 \mathrm{~ms}$. Thus, these stimulus parameters were used when the CNAP was not observed on each stimulus. In every case, at least 2 digital or interdigital nerves in the medial plantar nerve and one digital or interdigital nerve in the lateral plantar nerve were tested.

64 to 256 stimuli were averaged with a signal averager in each recording. When no recognisable and constant potential was noted after 256 stimuli had been averaged in three recordings, we concluded that there was no CNAP. Surface skin temperature under the arched portion of the medial plantar surface of the foot was controlled at $32^{\circ} \mathrm{C}$ using a skin temperature control unit. Latency was measured from the stimulus onset to the first positive peak for the maximum nerve conduction velocity (NCV), to the highest negative peak for the negative peak NCV, and to the last negative peak of the potential for the minimum NCV. Conduction velocity was calculated by dividing the distance by the latency. Distance was measured by caliper. The amplitude was measured from peak to peak. The duration was measured from the onset of the first positive peak to the baseline return of the last component of the potentials.

When the NCVs and the duration of CNAP in patients deviated by more than 2 standard deviations from the normal mean values, they were considered abnormal. Abnormally prolonged duration of CNAP is termed "dispersion phenomenon". When the amplitude of CNAP was lower than the lowest normal range, it was considered abnormal.

The motor terminal latency of the medial and lateral plantar nerves was determined following the method pre- 
Table 1 Sensory nerve conduction abnormalities in 25 cases of tarsal tunnel syndrome

\begin{tabular}{lcc}
\hline Plantar nerve & Medial & Lateral \\
\hline Normal & $1^{*}$ & 1 \\
Abnormal & 24 & 13 \\
Absent potential & 2 & 6 \\
Slow NCV & 16 & 4 \\
$\quad$ Maximum & 13 & 3 \\
Negative peak & 14 & 3 \\
Minimum & 10 & 1 \\
Dispersion & 14 & 5 \\
Low amplitude & 10 & 5 \\
\hline
\end{tabular}

* Number of cases

viously described. ${ }^{16}$ The distance between the recording electrodes and the stimulating electrodes was kept constant: $10 \mathrm{~cm}$ in the medial and $12 \mathrm{~cm}$ in the lateral plantar nerve.

Twenty-five cases of tarsal tunnel syndrome were diagnosed in 21 patients, 11 male and 10 female. Four patients

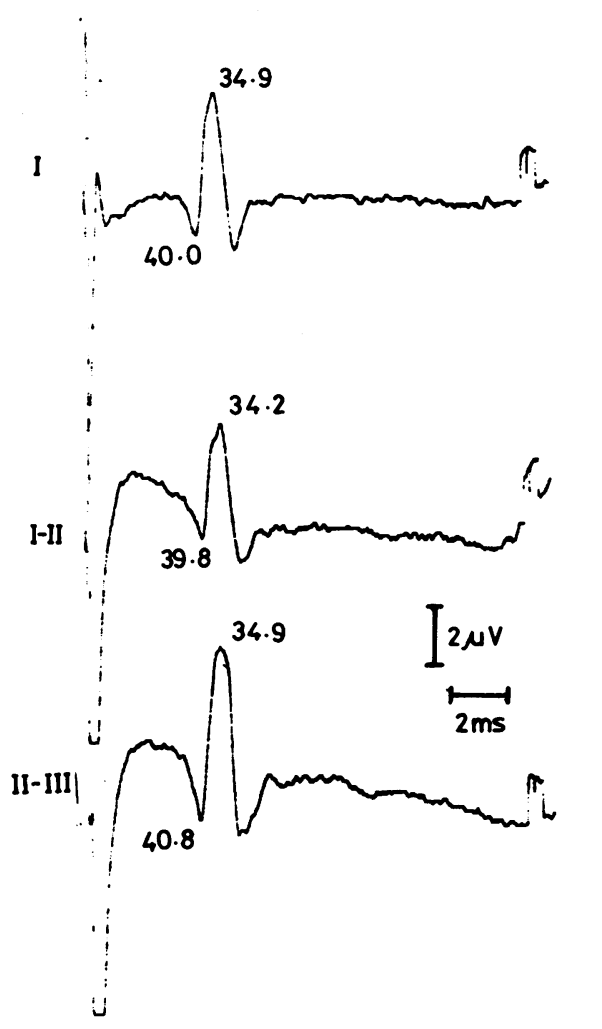

had bilateral tarsal tunnel syndrome. The ages of patients ranged from 14 to 69 years. Clinically, medial plantar neuropathy was diagnosed in 10 patients, lateral plantar neuropathy in two, and calcarine neuropathy in one. None of the patients had any motor weakness or wasting of the intrinsic foot muscles.

In all cases, subjective burning and tingling paraesthesia over the medial or lateral plantar nerve territory was present. For the diagnosis of tarsal tunnel syndrome, objective sensory impairment over the medial or lateral plantar nerve territory and a positive Tinel's sign on these nerves at the ankle were present in 20 cases; subjective symptoms and a Tinel's sign in three cases; and subjective symptoms and objective sensory impairment in two cases.

Lumbar radiculopathy and peripheral neuropathy were ruled out by neurological and electrophysiological evaluations when clinically indicated.

\section{Results}

Among the 25 cases of tarsal tunnel syndrome,

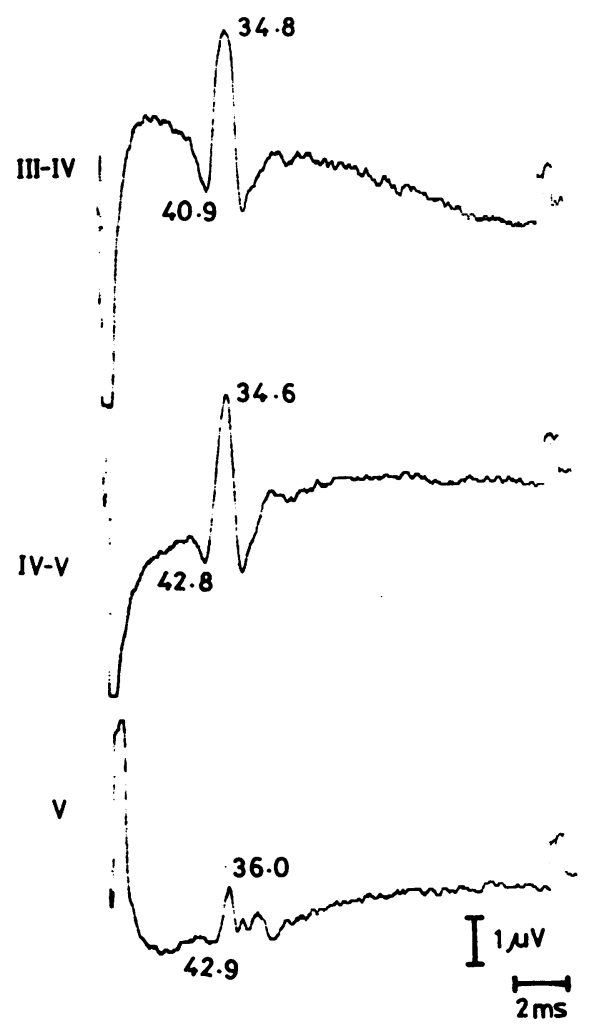

Fig 1 Normal maximum and negative-peak nerve conduction velocity (NCV) in a 29-year-old normal control. Roman numerals indicate digital and interdigital nerves. Numbers under the compound nerve action potentials (CNAP) represent maximum NCVs and numbers above the CNAPs represent negative-peak NCVs respectively. Two $\mu V$ calibration for the I and I-II interdigital nerves and $1 \mu V$ calibration for all other nerves. 


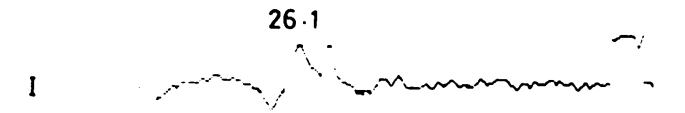

30.7
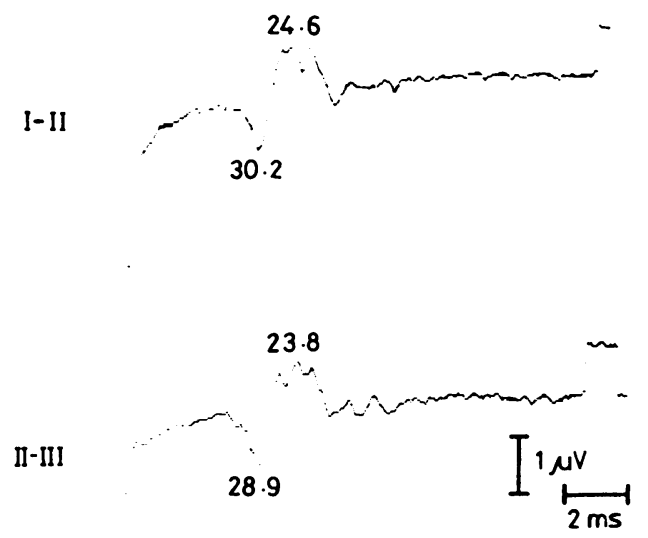
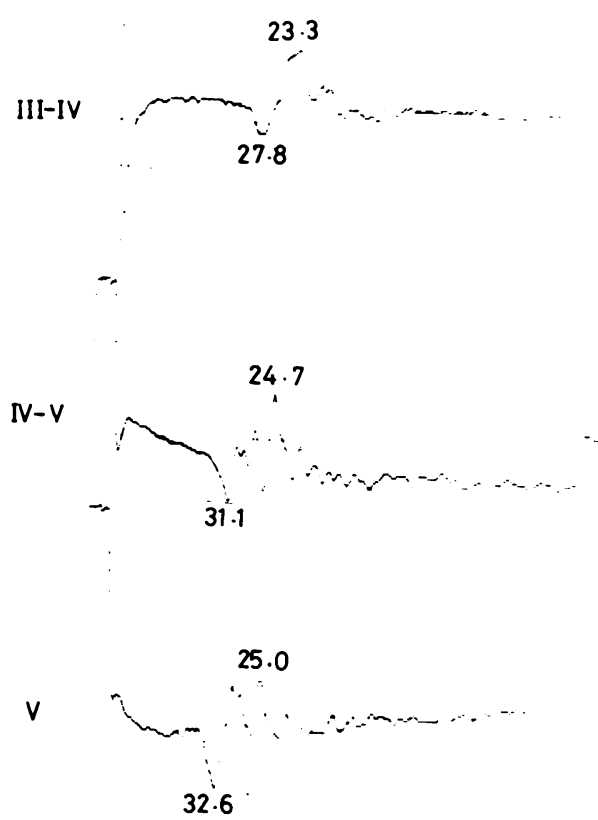

Fig 2 Slow maximum and negative-peak NCVs in medial plantar nerve and normal maximum and negative-peak NCVs in lateral plantar nerve in a case of tarsal tunnel syndrome.

abnormal sensory nerve conduction was observed in 24 (96\%): abnormal sensory nerve conduction in the medial plantar nerve in 24 cases and in the lateral plantar nerve in 13 cases (table 1). Thus, diagnosis was confirmed electrophysiologically in $96 \%$ of the cases of tarsal tunnel syndrome.

In 18 cases, various combinations of sensory conduction abnormalities (for example, slow NCV and dispersion phenomenon) were noted. In seven cases, a single sensory nerve conduction abnormality was noted: low amplitude in three, slow negative-peak $\mathrm{NCV}$ in two, dispersion phenomenon in two, and absent CNAPs in two.

Slow nerve conduction velocities (NCVs) and dispersion phenomenon were the most prominent abnormalities (figures 1-3). In 12 cases, slowing was observed both in maximum and negative-peak NCVs. In two cases, negative-peak NCV alone was slow, emphasising the value of the negative-peak NCV. Minimum NCV was slow in two cases in which maximum and negative-peak NCVs were normal but dispersion phenomenon was present. Thus, minimum NCV alone was not helpful in improving diagnostic sensitivity. Dispersion phenomenon and slow NCVs were present in 12 cases. In two cases, dispersion phenomenon was a single abnormality in sensory nerve conduction.
Mean values of the various nerve conduction parameters are presented in table 2. Maximum and negative-peak NCVs and duration of CNAP in the various interdigital nerves in tarsal tunnel syndrome are significantly different from normal values. However, the amplitudes of CNAP are only significantly lower than normal values in the I digital and I-II interdigital nerves. Slowing in minimum NCVs is barely significant in the I, II-III, and V nerves. ( $p<$ 0.05 )

Terminal latency of the plantar nerves was prolonged in only four of 24 tested cases (17\%): one in the medial plantar nerve $(<5.38 \mathrm{~ms})$ and three in the lateral plantar nerve $(<6.26 \mathrm{~ms})$ Mean terminal latencies were $4.00 \pm 0.77$ (SD) ms (vs. normal, $4 \cdot 10 \pm 0.64)$ in the medial plantar nerve and $4.81 \pm$ $1.06 \mathrm{~ms}$ (vs. normal, $4.70 \pm 0.78$ ) in the lateral plantar nerve. The amplitude of the compound muscle action potential was abnormally low in one case in the medial plantar nerve $(<3,500 \mu \mathrm{V})$, but none in the lateral plantar nerve $(<3,000 \mu \mathrm{V})$. Thus, motor nerve conduction was abnormal in $21 \%$ of tarsal tunnel syndrome cases.

\section{Discussion}

The tarsal tunnel syndrome is an entrapment 
30.8

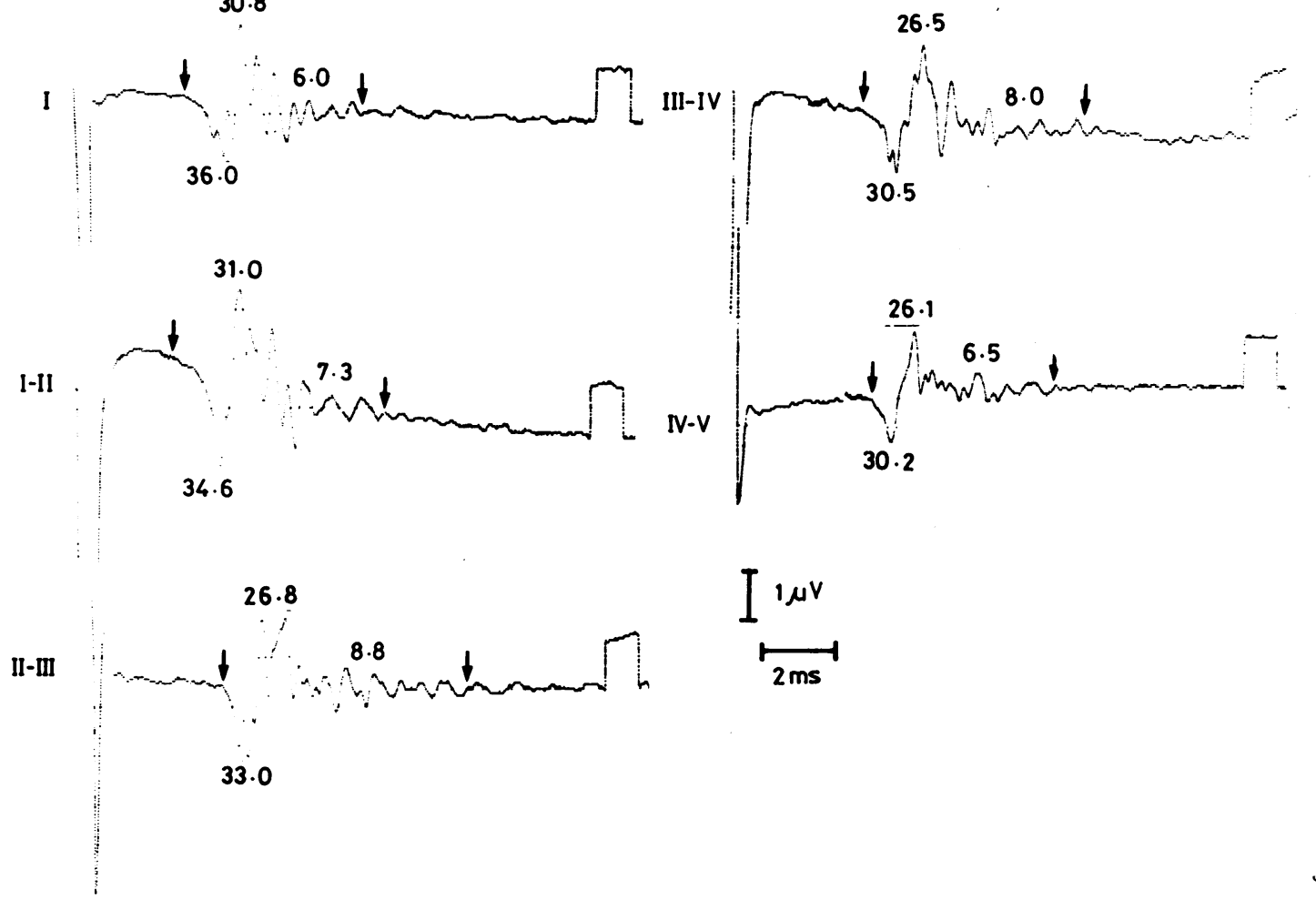

Fig 3 Dispersion phenomenon (prolonged duration of CNAPs) in I, I-II, II-III, and III-IV digital and interdigital nerves in a case of tarsal tunnel syndrome. Maximum and negative-peak NCVs are normal. Numbers between two arrows represent duration of CNAPs in ms.

Table 2 Mean sensory nerve conduction values in plantar nerves in 25 cases of tarsal tunnel syndrome

\begin{tabular}{|c|c|c|c|c|c|}
\hline & \multicolumn{3}{|c|}{ Nerve conduction velocities } & \multirow{2}{*}{$\begin{array}{l}\text { Duration } \\
\text { (ms) }\end{array}$} & \multirow{2}{*}{ Amplitude } \\
\hline & Maximum & Negat peak $(\mathrm{m} / \mathrm{s})$ & Minimum & & \\
\hline $\begin{array}{l}I \\
(N=24) \\
I-I I \\
(N=22) \\
\text { II-III } \\
(N=17) \\
\text { III-IV } \\
(N=14) \\
I V-V \\
(N=20) \\
(N=5) \\
(N=5)\end{array}$ & $\begin{array}{c}32.8 \pm 4.1^{*} \\
(40.5 \pm 2.7) \dagger \\
32.6 \pm 4.38 \\
(38.4 \pm 2.9) \\
29.7 \pm 4.88 \\
(36.3 \pm 3.2) \\
31.7 \pm 4.7 \pm \\
(35.9 \pm 3.2) \\
32.1 \pm 5.48 \\
(37.2 \pm 2.7) \\
31.3 \pm 2.8 \pm \\
(38.4 \pm 4.0)\end{array}$ & $\begin{array}{r}27.7 \pm 3.88 \\
(34.8 \pm 2.2) \\
27.1 \pm 3.78 \\
(33.0 \pm 2.4) \\
25.9 \pm 3.78 \\
(31.3 \pm 2.6) \\
25.6 \pm 3.88 \\
(30.7 \pm 2.6) \\
27.4 \pm 4.08 \\
(32.2 \pm 3.4) \\
26.4 \pm 3.0 \pm \\
(32.3 \pm 3.2)\end{array}$ & $\begin{array}{c}17.9 \pm 3.2 \\
(23.0 \pm 3.3) \\
17.5 \pm 3.8 \\
(21.4 \pm 3.1) \\
15.8 \pm 3.1 \\
(20.2 \pm 3.2) \\
17.4 \pm 4.2 \\
(20.1 \pm 3.0) \\
17.6 \pm 3.8 \\
(20.8 \pm 3.7 \\
17.5 \pm 3.0 \\
(23.2 \pm 3.0)\end{array}$ & $\begin{array}{r}6.0 \pm 2.48 \\
(3.2 \pm 1.0) \\
5.7 \pm 2.18 \\
(3.8 \pm 1.1) \\
6.3 \pm 1.68 \\
(4.2 \pm 1.2) \\
6.2 \pm 1.98 \\
(3.8 \pm 1.1) \\
5.4 \pm 2.08 \\
(3.7 \pm 1.2) \\
5.3 \pm 3.5 \pm \\
(3.0 \pm 0.9)\end{array}$ & $\begin{array}{r}2.4 \pm 2.78 \\
(5.5 \pm 1.7) \\
3.1 \pm 3.1 \neq \\
(5.4 \pm 1.7) \\
3.1 \pm 3.8 \\
(3.9 \pm 1.8) \\
2.7 \pm 3.4 \\
(3.4 \pm 1.4) \\
2.0 \pm 2.4 \\
(2.8 \pm 1.2) \\
1.4 \pm 0.9 \\
(1.8 \pm 0.9)\end{array}$ \\
\hline \multicolumn{6}{|c|}{$\begin{array}{l}\text { I-V refers to the interdigital nerves. } \\
\text { N: Number of cases. } \\
\text { "Mean } \pm \text { standard deviation. } \\
\text { tNormal values for } 20-49 \text {-year-old group } \\
\text { fDifference is significant from normal values (for } 20-49 \text {-year-old group) at } p<0.01 \text {. } \\
\text { \&Difference is significant at } p<0.001 \text {. }\end{array}$} \\
\hline
\end{tabular}


neuropathy of the posterior tibial nerve and its branches within the fibro-osseous tunnel that lies beneath the flexor retinaculum on the medial side of the ankle. The most prominent symptom is burning and tingling paraesthesia in the toes and along the sole of the foot. These symptoms are increased by activity. The most helpful objective findings in diagnosis are a positive Tinel's sign in the posterior tibial nerve at the ankle and sensory impairment in the plantar nerve territory.'

It is important to differentiate tarsal tunnel syndrome from distal sensory neuropathy. The most helpful clinical signs indicative of sensory neuropathy are an objective "stocking distribution sensory loss" on the foot, including the dorsum, and absent Tinel's sign at the posterior tibial nerve at the ankle. In tarsal tunnel syndrome, there should not be any objective sensory loss over the dorsum of the foot. To rule out sensory neuropathy which can mimic tarsal tunnel syndrome, we performed motor nerve conduction studies in the posterior tibial and peroneal nerves and sensory nerve conduction in the sural nerve when clinical features were atypical. In all cases tested, they were normal.

Until 1979, when we introduced the sensory nerve conduction technique with a surface electrode in the medial and lateral plantar nerves, ${ }^{\prime}$ prolonged terminal latency of the medial or lateral plantar nerve was used as an objective diagnostic criterion for tarsal tunnel syndrome. ${ }^{3-5}$ However, the diagnostic sensitivity of the terminal latency was low, the terminal latency being prolonged in only $54 \%$ of cases.' In contrast, sensory nerve conduction studies with the surface electrode was far superior in confirming the diagnosis of tarsal tunnel syndrome, being abnormal in $90.5 \%$ of cases. The most common sensory nerve conduction abnormality with the surface electrodes was absent CNAP, as observed in the medial plantar nerve in $68 \%$ and in the lateral plantar nerve in $83 \%$ of abnormal cases.

The superiority of the near-nerve needle technique over the surface electrode in study of sensory nerve conduction is well-known. ${ }^{6}$ The present study confirmed such findings. With the near-nerve needle technique, we were able to record CNAPs in a majority of patients. CNAPs were absent in only $8 \%$ of abnormal cases in the medial plantar nerve and in $46 \%$ in the lateral plantar nerve. With the nearnerve needle technique, dispersion phenomenon was also recorded and diagnostic sensitivity was improved from $90.5 \%$ to $96 \%$.

On the other hand, the diagnostic sensitivity of the terminal latency for tarsal tunnel syndrome was $17 \%$, far lower than our previously reported figure $(54 \%) .^{1}$ The lower figure in this study reflects the milder nature of the disorder in the present series.
Our study clearly shows that the near-nerve sensory nerve conduction study is far superior objective diagnostic index of tarsal tunnel syndrome than the terminal latency test. Compared with carpal tunnel syndrome, in which terminal latency is prolonged in $61-91 \%$ of cases, ${ }^{6}$ terminal latency measurement is less helpful in the diagnosis of tarsal tunnel syndrome. The reason for this discrepancy is not apparent. Seddon stated that the tarsal tunnel differs from the carpal tunnel in that the flexor retinaculum is thinner. ${ }^{7}$ One may speculate that the thinner flexor retinaculum renders the nerve less compressible in the tarsal tunnel than in the carpal tunnel, producing a "milder form of entrapment neuropathy" in tarsal tunnel syndrome and making the terminal latency less helpful in its diagnosis.

The most important finding in the present study is the elucidation of the basic pathological process in tarsal tunnel syndrome. We have documented that slow NCV and dispersion phenomenon are the most prominent abnormalities in sensory nerve conduction in the syndrome. These two abnormalities are the electrophysiological hallmarks of a neuropathy with predominant segmental demyelination. ${ }^{8}$ Thus, it can be concluded that focal segmental demyelination is the primary pathological process in tarsal tunnel syndrome. This finding is consistent with the well-known pathology of entrapment neuropathies, with complete segmental demyelination in advanced cases. ${ }^{9}$

\section{References}

${ }^{1}$ Oh SJ, Sarala PK, Kuba T, Elmore RS. Tarsal tunnel syndrome: electrophysiological diagnosis. Ann Neurol 1979;5:327-30.

${ }^{2}$ Oh SJ, Kim HS, Ahmad BK. Electrophysiological diagnosis of interdigital neuropathy of the foot. Muscle Nerve 1984;7:218-25.

${ }^{3}$ Goodgold J, Kopell HP, Spielholz NI. The tarsal tunnel syndrome. N Eng J Med 1965;273:742-5.

${ }^{4}$ Johnson EW, Ortiz PR. Electrodiagnosis of tarsal tunnel syndrome. Arch Phys Med Rehabil 1966;47:776-80.

5 Mann RA. Tarsal tunnel syndrome. Orthop Clin North Am 1974;5:109-15.

- Oh SJ. Clinical Electromyography: Nerve Conduction Studies. Baltimore: University Press, 1984.

7 Seddon H. Surgical Disorders of the Peripheral Nerves. 2nd ed. Edinburgh: Churchill Livingstone, 1975:126.

${ }^{8}$ Buchthal F, Rosenfalck A, Behse F. Sensory potentials of normal and diseased nerves. In: Dyck PJ, Thomas PK, Lambert EH, eds. Peripheral Neuropathy, vol 1. Philadelphia: WB Saunders Co, 1975:442-64.

9 Gilliatt RW. Chronic nerve compression and entrapment. In: Sumner, AJ, ed. The Physiology of Peripheral Nerve Disease. Philadelphia: WB Saunders Co, 1980:316-39. 